REVIEW

\title{
Under-recognised paradox of neuropathy from rapid glycaemic control
}

\section{K S Leow, J Wyckoff}

Postgrad Med J 2005;81:103-107. doi: 10.1136/pgmi.2004.021162

Insulin induced neuropathy has been reported previously in people with diabetes treated with insulin, and subsequently reported in patients with insulinomas. However, neuropathy caused by rapid glycaemic control in patients with poorly controlled diabetes with chronic hyperglycaemia is not a widely recognised entity among clinicians worldwide. It is expected that this phenomenon of paradoxical complication of neuropathy in the face of drastic decreases in glycosylated haemoglobin concentrations will assume greater importance with clinicians achieving glycaemic targets at a faster pace than before.

See end of article for authors' affiliations ....................

Correspondence to: Dr M K S Leow Department of Endocrinology, Division of Medicine, Tan Tock Seng Hospital, Singapore 308433; mleowsj@ massmed.org

Submitted 25 February 2004 Accepted 31 May 2004
D iabetic neuropathy is an established microvascular complication related to suboptimal glycaemic control. But hyperglycaemia is not the sole factor in the pathogenesis of neuropathy in people with diabetes. ${ }^{1}$ Scattered throughout the medical literature are sporadic reports of deterioration of neuropathy that apparently occurred during dramatic resolution of chronic hyperglycaemia, associated with precipitous declines in glycosylated haemoglobin (HbAlc) concentrations. ${ }^{23}$ This unexpected phenomenon implicates hypoglycaemia in the precipitation of neuropathy in both patients with pre-existing neuropathy and normal baseline neurological status, given that those without a history of neuropathy develop it after they become afflicted with insulinomas. ${ }^{4}$ It is debatable as to whether the mere rapid attainment of euglycaemia, associated with drastic decreases in HbAlc concentrations without hypoglycaemic episodes punctuating the clinical course, can result in this complication likewise. While the results are being unravelled, it is prudent to instil in clinicians the awareness of such entities and emphasise the dual edged sword of rapid normalisation of chronically raised blood glucose concentrations as the universal strive to reach satisfactory glycaemic end points continues.

\section{EPIDEMIOLOGY AND NATURAL HISTORY} Strength of correlation of neuropathy with rapid glycaemic control

"Insulin neuritis" surfaced after the dawn of insulin therapy back in the first quarter of the past century, and represented the earliest report of neuropathy accompanying rapid reversal of hyperglycaemia. $^{5-8}$ This is usually a distal sensory mechanisms of such seemingly paradoxical polyneuropathy developing within a month of aggressive diabetic control with intensive insulin therapy. In time, reports of similar peripheral neuropathy were also found in patients with insulinomas. ${ }^{910}$ Enigmatically, this complication has yet to be reported with comparable frequency in those treated with oral antidiabetic agents. It is indeed possible that other forms of neuropathy such as diabetic autonomic neuropathy could also be worsened or precipitated by acute reversal of chronic hyperglycaemia, but this possibility will only become apparent as more cases are reported in the literature. ${ }^{11}$

Scale of impact of paradoxical neuropathy induced by diabetes treatment

Neuropathy secondary to rapid normalisation of chronic hyperglycaemia in the setting of poorly controlled diabetes is insidiously emerging as a new disease entity classifiable as an iatrogenic complication. Despite the paucity of large scale systematic studies, this form of neuropathy could merely represent the tip of the "iceberg". Perhaps, the full impact of what appears to be a rare complication will be better appreciated globally through heightened awareness. Although the magnitude of neuropathy induced by diabetes therapy has not been determined by large scale studies, early worsening of diabetic retinopathy as a model of microvascular complication subsequent to treatment of diabetes has been well reported in clinical trials. ${ }^{12}$ Notably, the first report of early deterioration of a diabetic microvascular end point resulting from improved glycaemic control was focused on retinopathy, and came from the authoritative diabetes control and complications trial (DCCT) in which $22 \%$ of study subjects in the intensive insulin therapy arm and $13 \%$ of those in the conventional therapy group experienced significant deterioration of diabetic retinopathy in the initial phase of improvement in glycaemic control..$^{12}{ }^{13}$ However, it was reassuring to note that the deterioration of retinopathy was transient and seems to stabilise and resolve with persistent attainment of euglycaemia after 18 months of insulin therapy. ${ }^{12}{ }^{13}$ Whether neuropathic deterioration behaves likewise remains unknown. Thus far, clinicians mainly realise that optimal glycaemic control results in a significant reduction in serious long term neuropathy in type 1 diabetes mellitus by $69 \%$ as conclusively shown in the landmark

Abbreviations: $\mathrm{HbAlc}$, glycosylated haemoglobulin; DCCT, diabetes control and complications trial; UKPDS, United Kingdom prospective diabetes study; HIF- $1 \alpha$, hypoxia inducible transcription factor- $1 \alpha$; HIF- $2 \alpha$, hypoxia inducible transcription factor- $2 \alpha$ 
DCCT and mirrored by the United Kingdom prospective diabetes study (UKPDS) of patients with type 2 diabetes mellitus. ${ }^{13}{ }^{14}$ It would require long term trials with adequate statistical power to evaluate neuropathy triggered by restoration of euglycaemia for evidence based guidelines to be formulated that incorporate the recommendations of both the DCCT and UKPDS without tipping the benefit to risk ratio unfavourably.

\section{JUSTIFICATION OF PRESENT CONCERNS OF RAPID ACHIEVEMENT OF EUGLYCAEMIA AS "NON- TRADITIONAL" NEUROPATHY RISK FACTOR Extent of physicians following evidence based glycaemic targets to avoid chronic diabetic complications}

Even if the observed association between drastic declines in chronically increased HbAlc and precipitation of neuropathy were truly causal, its importance will only draw clinical attention when a critical mass of physicians worldwide have significantly changed their practices by achieving the desired glycaemic goals much more than they ever did in the past, and at an inordinately swifter pace than before. If this supposition were correct, the process of rapid attainment of glycaemic goals in poorly controlled diabetics could then transform into a paradoxical "non-traditional" risk factor for neuropathy.

For an unbiased notion of the extent of change in clinical practices, it is instructive to consider some pertinent comparative studies done on diabetic control both in the before and after DCCT/UKPDS era. Bradshaw et al reported a large proportion of both types 1 and 2 diabetic patients between 1983 and 1992 had HbAlc above those associated with increased risk of microvascular complications. ${ }^{15}$ However, recent investigators showed that the DCCT defined new standards of care not rigorously pursued before the inception of the study. For instance, longitudinal HbAlc values of 884 subjects with type 1 diabetes from 1993 to 1998 declined significantly after the DCCT report. ${ }^{16}$ Moreover, the introduction of rapid insulin analogues such as insulin lispro from 1996 saw yet another rapid decline in HbAlc values beyond the reduction post-DCCT. ${ }^{16}$ A multicentre epidemiological survey on type 2 diabetics showed that the mean HbAlc value in the post-DCCT years is lower than in prior surveys and suggested that HbAlc values are plummeting downwards. ${ }^{17}$ This accelerated impetus to attain stringent glycaemic targets cuts both ways, as its practice is evidence based and yet it potentially fuels aggressive glycaemic control without due consideration to what might constitute a reasonably safe speed of achieving euglycaemia.

\section{Awareness among physicians of the deleterious effects of rapid glycaemic control on neuropathy} It is common knowledge that a "learning curve" generally influences the incidence of complications for any new medical intervention. Unfortunately, it is not clear exactly what proportion of physicians treating diabetics are actually aware of the possibility of causing potential harm to nerve function as a complication of intensified glycaemic control. While evidence from studies such as the DCCT and UKPDS form the basis of daily clinical practice, it is still largely oblivious to clinicians that highly intensive treatment that rapidly achieves glycaemic targets may ironically be undesirable and even have deleterious impacts on microvascular outcomes. This is because neuropathy has been so well established as a known microvascular complication of poorly controlled diabetes that it becomes counter intuitive to imagine that rapid glycaemic control should result in the very form of complication that good control is supposed to prevent. Because of the apparent rarity of this perplexing phenomenon, there is a dearth of information written about it. Even more esoteric than peripheral neuropathy is autonomic neuropathy that is worsened by rapid glycaemic control. ${ }^{11}$ This implies that healthcare providers would probably benefit from better dissemination of such knowledge. On the same token, it is also important that hypoglycaemic neuropathy of indeterminate incidence not be hyped to the extreme as to create unnecessary confusion and therapeutic quandary that undermine on existing standards of care in line with DCCT/UKPDS based guidelines verified to reduce diabetic microvascular end points. The desirable stance is a balanced viewpoint such that physicians appreciate treatment related neuropathy as a distinct entity and consequently prevent this complication in future with better characterisation and understanding of this morbidity. In many ways, reinforcing such "diabetic rarities" can modify the knowledge, attitudes, and practices of physicians, which can pave the way for both improved diagnosis and establishment of new standards of care for people with diabetes. By then, it behoves the practising physician to strive for better glycaemic control in diabetics without incurring excess risk of precipitating neuropathy.

\section{BIOLOGICAL BASIS}

\section{Neurotoxicity of hypoglycaemia at the cellular and molecular level}

The nervous system uses glucose as its main immediate metabolic substrate for energy, and is therefore especially vulnerable to hypoglycaemia. Exogenous insulin has been shown to induce peripheral nerve lesions in animal models. ${ }^{18}$ When hypoglycaemia becomes profound and protracted, even neurons of the anterior horns of the spinal cord and dorsal root ganglia are not spared from injury. There is pronounced loss of anterior horn neurons via Wallerian degeneration and loss of large myelinated fibres in hypoglycaemic neuropathy, in stark contrast with diabetic neuropathy attributable to long term hyperglycaemia that is associated with striking axonal atrophy of chiefly sensory fibres without significant neuronal loss. ${ }^{19}$ Insulin induced hypoglycaemia causes predominantly central fascicular axonal degeneration of peripheral nerves. ${ }^{20}$ Although previous studies suggested neuronal death attributable to hypoglycaemia occurs by necrosis, more recent findings point to apoptosis as yet another molecular mechanism from the increased expression of Bax and caspase-like activation. ${ }^{21}{ }^{22}$ Hypoglycaemia was shown to directly cause axonal degeneration and indirectly result in neuronal necrosis through the action of excitatory amino acids on the $N$-methyl-D-aspartate (NMDA) receptor. ${ }^{23}$ However, this excitotoxin theory remains conjectural as excitotoxic amino acids are better established to target destruction of neuronal cell bodies and dendrites more so than axons. ${ }^{24}$ Whether cytokines participate and contribute to the neurotoxicity of hypoglycaemia is still not well clarified.

It is debatable whether hypoglycaemia itself, or hyperinsulinaemia, or both contribute to neuropathy. Sugimoto et al discovered that hypoglycaemia in rats is associated with increased myelinated axonal damage, while hyperinsulinaemia is associated with increased densities of small myelinated axons and endoneurial microvessels with microangiopathic changes. ${ }^{25}$ In a cross sectional population study that compared the prevalence of neuropathy and retinopathy in type 2 diabetics treated with insulin versus sulfonylureas, multivariate analysis showed that the frequency of neuropathy was significantly higher in the insulin group $(63 \%$ versus $49 \%$; $p<0.016)$ as was retinopathy ( $85 \%$ versus $58 \%$; $\mathrm{p}<0.0001)$. This difference was attributed to insulin being more mitogenic, atherogenic, and thrombogenic as compared with sulfonylureas. ${ }^{26}$ 


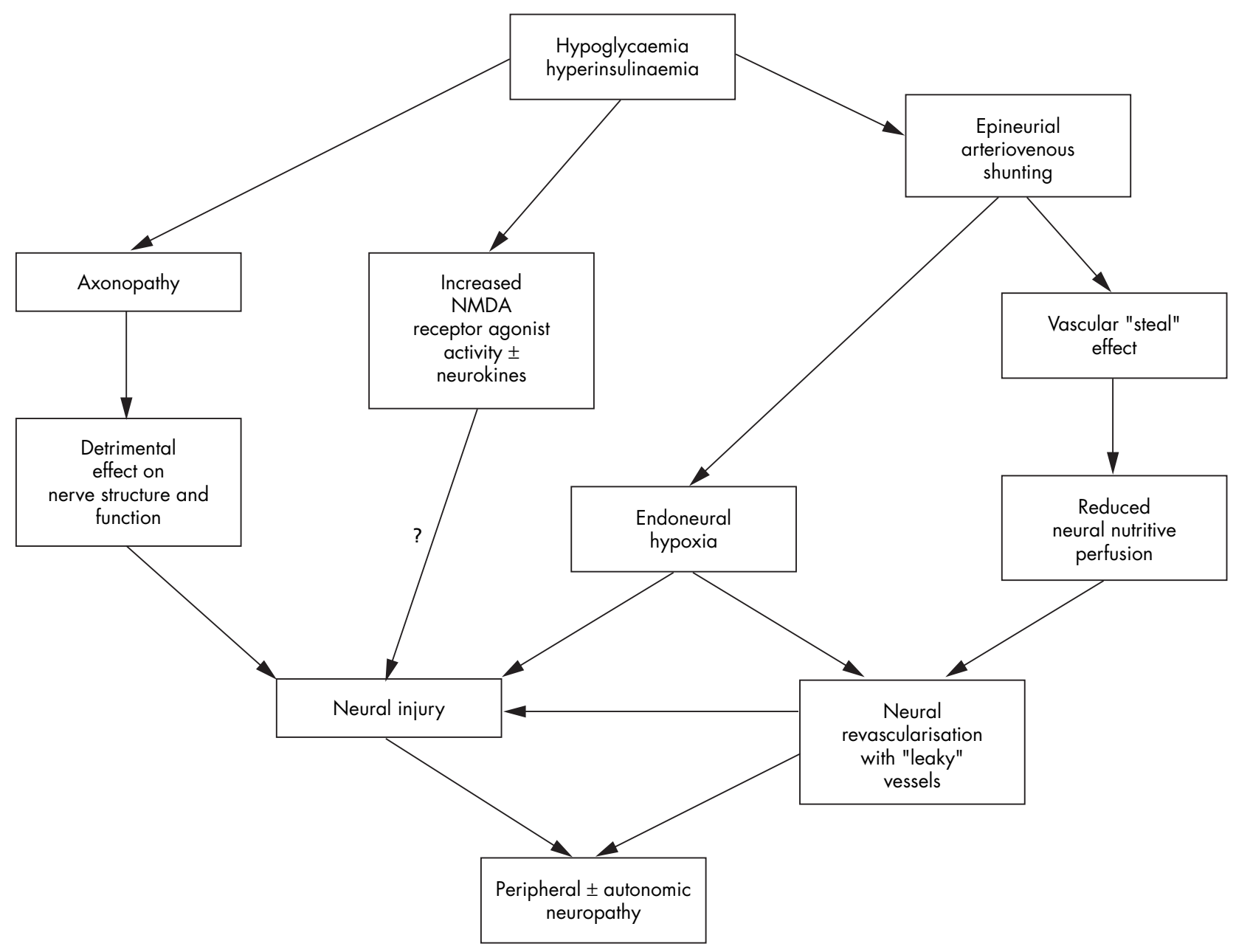

Figure 1 Proposed mechanisms of neuropathy precipitated by rapid glycaemic control.

\section{Proposed pathophysiological mechanisms}

Experimental evidence points to hypoglycaemia rather than hyperinsulinaemia, as the pathogenetic cause of reduction in the fast anterograde axonal transport. ${ }^{27}$ Mandelbaum et al showed through regression analysis that the degree of axonal degeneration bears a significant inverse correlation to the glucose levels, and supports an association between axonal degeneration and the episodes of hypoglycaemia. ${ }^{28}$ This probably represents the underlying pathophysiological basis of neuropathy precipitated by sharp declines in HbAlc attributable to multiple hypoglycaemic spells. Experiments on murine models showed that acute hypoglycaemia attenuates neural perfusion to almost $40 \%$ of controls. ${ }^{29}$ A further advance came about with the elucidation of endoneurial hypoxia directly mediated by insulin (endogenous or exogenous) through increased arteriovenous flow and simultaneous reduced nutritive flow of nerves. Yet there is a resistance to this hypoxia that stems from increased energy substrate stores in the diabetic state coupled with low energy requirement of peripheral nerves. With antidiabetic therapy, the energy substrates are normalised and the susceptibility to endoneurial hypoxia returns to trigger nerve fibre degeneration. ${ }^{11}{ }^{30}$ Recently, hypoxia was found to trigger apoptosis mediated via hypoxia inducible transcription factor- $1 \propto$ (HIF$1 \alpha){ }^{31}{ }^{32}$ Another fascinating development was the finding that hypoxia inducible transcription factor- $2 \alpha$ (HIF- $2 \alpha$ ) plays a part in the cellular apoptosis attributable to hypoglycaemia but not to hypoxia. ${ }^{33}$ Through HIF- $1 \alpha$ and HIF- $2 \alpha$ acting on hypoxia response elements, hypoglycaemia and hypoxia probably regulate genes governing apoptosis and survival such as p53 and Bcl-2. Thus, current concepts support acute neural vascular insufficiency and metabolic dysequilibrium in the precipitation and aggravation of neuropathy by rapid glycaemic control, and may be summarised schematically as shown in figure 1 .

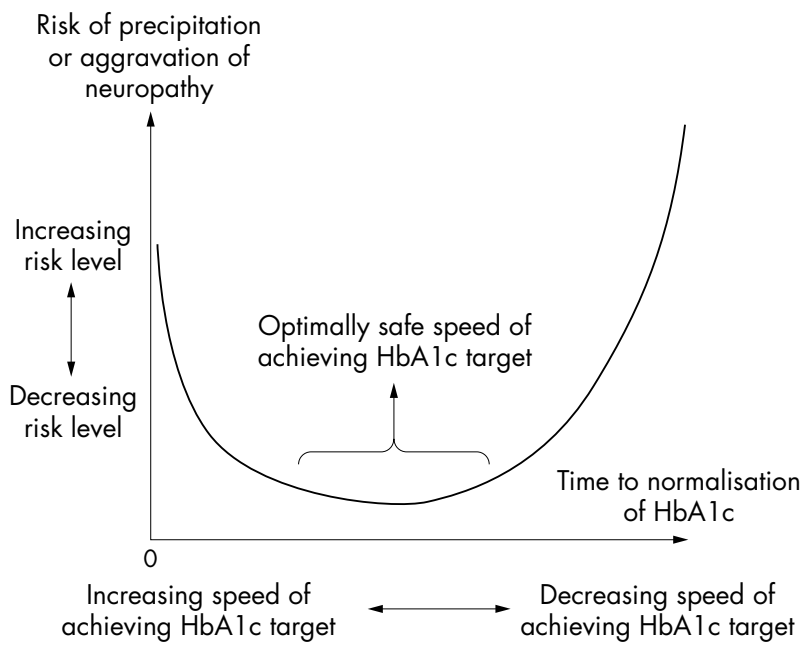

Figure 2 Hypothetical relation of risk of neuropathy with rate of glycaemic control. 


\section{CLINICAL MANIFESTATION AND DIAGNOSIS Clinical observations}

Patients with insulinomas suffering from hypoglycaemic polyneuropathy usually present with symmetric weakness and distal wasting of hands and feet, absent tendon reflexes, and "glove and stocking" loss of sensation or paraesthesia. ${ }^{34}$ Upper extremity involvement, in particular atrophy of the thenar, hypothenar, and interossei, muscles, is more frequent. $^{35}{ }^{36}$ Painful distal paraesthesias without objective sensory loss are typical. Similar acute and painful polyneuropathy has been reported in people with diabetes who experienced precipitous declines in HbAlc. ${ }^{3}$ When the feet are affected, severe pain may impair ambulation. Fasciculations are absent, and electromyographic studies show denervation action potentials with reduction of nerve conduction velocities, consistent with sensorimotor peripheral neuropathy. ${ }^{10}$ Muscle action potential amplitudes of motor nerves are diminished, and sensory action potentials are absent. Hence, the evidence suggests that axonal sensorimotor neuropathy characterises hypoglycaemic neuropathy. ${ }^{37}$ Symptoms may improve with time as euglycaemia is maintained, thus showing that the prognosis for recovery may be favourable. However, it remains unknown if complete neurological recovery will occur in every affected case.

\section{Paucity of the existing clinical literature}

Whereas many reports cited insulinomas and exogenous insulin therapy as the main culprits in inducing neuropathy, there are virtually no case reports in the existing literature to date after an extensive Medline search of similar hypoglycaemic sensorimotor polyneuropathy triggered by endogenous insulin excess attributable to oral antidiabetic agents (for example, insulin secretagogues such as sulfonylureas, meglitinides) or hypoglycaemia induced by non-insulin mediated mechanisms such as certain forms of tumours secreting IGF2. The same can be said for abrupt restoration of euglycaemia by pancreatic or islet transplantation. Only an isolated case report was found for hypoglycaemic neuropathy caused by recurrent hypoglycaemic spells attributable to anti-insulin receptor antibodies. ${ }^{38}$ Hence, apart from insulinomas and insulin therapy, all the other potential aetiologies at best represent only theoretical risks, and at worst await future confirmation of their harm through better recognition of the syndrome. For the present, the paucity of data limits any attempted appraisal on the correlation of precipitation of neuropathy by rapid glycaemic control by oral agents or pancreatic transplantation. Given that the weight of the evidence points to hypoglycaemia rather than hyperinsulinaemia as the primary causative factor in the genesis of hypoglycaemic sensorimotor polyneuropathy, it is therefore entirely plausible that repeated hypoglycaemic insults attributable to causes other than insulinoma or exogenous insulin can result in the same complication.

\section{Learning points}

- For patients with a brief history of poorly controlled diabetes, the risk versus benefit analysis overwhelmingly favours rapid glycaemic control.

- In diabetic patients with chronically poor glycaemic control, with the exceptions of hyperglycaemic emergencies such as diabetic ketoacidosis or hyperglycaemic-hyperosmolar non-ketototic coma, it is prudent to attain their glycaemic targets over a gradual time frame to avoid acute deterioration and precipitation of retinopathy and neuropathy.

\section{SUMMARY OF PERSPECTIVES AND FUTURE DIRECTIONS}

It is expected that a wider circle of clinicians will gradually appreciate the risk of precipitation of neuropathy in people with diabetes attributable to disproportionately rapid glycaemic control. Acknowledgement of this risk by experts can in turn assist the development of position statements and safety guidelines on the appropriate pace that glycaemic control should be brought about in poorly controlled diabetics. Efforts to prevent this complication should however be balanced by a purposeful drive to lower HbAlc values to desirable targets over reasonable timeframes. For now, it is timely to put in perspective a hypothetical "U shaped" correlation of risk of development of neuropathy with the speed of achievement of the desired HbAlc targets in diabetic patients as shown in figure 2 . This implies the existence of a certain optimal rate of achieving the glycaemic control such that the overall risk of neuropathy is at a minimum for any given diabetic patient. An important task and priority of future researchers will be to study and determine that optimal rate of glycaemic control that can lead to maximal reduction of the risk of development of microvascular complications and simultaneously minimising the risk of hypoglycaemic sensorimotor polyneuropathy.

\section{LESSONS LEARNED AND CONCLUDING REMARKS}

The risk of precipitation of hypoglycaemic neuropathy seems to be clinically significant as the rate of decline of HbAlc becomes inordinately rapid. The prognosis of neuropathy caused by overzealous antidiabetic therapy to achieve glycaemic targets remains speculative, although some afflicted cases have apparently made remarkable recovery. But such anecdotal cases may not serve well as prognostic yardsticks because total resolution could be limited if neuronal death is the predominant mechanism of the neuropathy.

\section{ACKNOWLEDGEMENTS}

We thank Jane Sim-Joo Tan and Rachel May-Wern Leow for their patience in proofreading the manuscript as well as their excellent editorial and secretarial assistance.

\section{Authors' affiliations}

M K S Leow, Department of Endocrinology, Division of Medicine, Tan Tock Seng Hospital, Singapore

J Wyckoff, Joslin Clinic, Joslin Diabetes Center, Harvard Medical School, USA

Funding: none.

Competing interests: none.

\section{REFERENCES}

1 Simmons Z, Feldman EL. Update on diabetic neuropathy. Curr Opin Neurol 2002; 15:595-603.

2 Llewelyn JG, Thomas PK, Fonseca V, et al. Acute painful diabetic neuropathy precipitated by strict glycemic control. Acta Neuropathol 1986;72:157-63.

3 Wilson JL, Sokol DK, Smith LH, et al. Acute painful neuropathy (insulin neuritis) in a boy following rapid glycemic control for type 1 diabetes mellitus. J Child Neurol 2003; 18:365-7.

4 Heckmann JG, Dietrich W, Hohenberger W, et al. Hypoglycemic sensorimotor polyneuropathy associated with insulinoma. Muscle Nerve 2000;23:1891-4.

5 Caravat CM. Insulin neuritis. A case report. Va Med Mo 1933;59:745-6.

6 Ellenberg M. Diabetic neuropathy precipitating after institution of diabetic control. Am J Med Sci 1958;236:466-71.

7 Wauchope GM. Critical review: hypoglycemia. Q J Med 1933:21:117.

8 Jordan WR. Neuritic manifestations in diabetes mellitus. Arch Intern Med 1936;57:307

9 Silfveskioild BP. "Polyneuritis hypoglycemia": late peripheral paresis after hypoglycemic attacks in two insulinoma patients. Acta Med Scand 1946; 125:502.

10 Jaspan JB, Wollman RL, Bernstein L, et al. Hypoglycemic peripheral neuropathy in association with insulinoma: implications of glucopenia rather 
than hyperinsulinism. Case report and literature review. Medicine 1982;61:33-44

11 Tesfaye S, Malik R, Harris N, et al. Arterio-venous shunting and proliferating new vessels in acute painful neuropathy of rapid glycemic control (insulin neuritis). Diabetologia 1996;39:328-35.

12 Diabetes Control and Complications Trial Research Group. The effect of intensive diabetes treatment on the progression of diabetic retinopathy on insulin-dependent diabetes mellitus. Arch Ophthalmol 1995; 113:36-51.

13 Diabetes Control and Complications Trial Research Group. The effect of intensive treatment of diabetes in the development and progression of longterm complications in insulin-dependent diabetes mellitus. N Engl J Med 1993;329:977-86.

14 UK Prospective Diabetes Study (UKPDS) Group. Intensive blood glucose control with sulphonylureas or insulin compared with conventional treatment and risk of complications in patients with type 2 diabetes (UKPDS 33). Lancet 1998;352:837-53.

15 Bradshaw C, Steen IN, Eccles M. Glycated haemoglobin levels in patients with diabetes in one general practice over a 10-year period. Diabet Med $1995 ; 12: 628-31$

16 Chase HP, Lockspeiser T, Peery B, et al. The impact of the diabetes control and complications trial and humalog insulin on glycohemoglobin levels and severe hypoglycemia in type 1 diabetes. Diabetes Care 2001;24:430-4.

17 Harmel AP, Ryan D, Thompson R. Glycohemoglobin assessment program: glycated hemoglobin and epidemiologic variables in patients with type 2 diabetes. Endocr Pract 2002;8:184-90.

18 Potter CG, Sharma AK, Farber MO, et al. Hypoglycemic neuropathy in experimental diabetes. J Neurol Sci 1988;88:293-301.

19 Sima AA, Zhang WX, Greene DA. Diabetic and hypoglycemic neuropathy-a comparison in the BB rat. Diabetes Res Clin Pract 1989;6:279-96.

20 Yasaki S, Dyck PJ. Spatial distribution of fiber degeneration in hypoglycemic neuropathy in rat. J Neuropathol Exp Neurol 1991;50:681.

21 Ouyang YB, He QP, Li PA, et al. Is neuronal injury caused by hypoglycemic coma of the necrotic or apoptotic type? Neurochem Res 2000;25:661-7.

22 Nath R, Probert A Jr, McGinnis KM, et al. Evidence for activation of caspase3 -like protease in excitotoxin and hypoxia/hypoglycemia injured neurons. J Neurochem 1998;71:186-95.

23 Wieloch T. Hypoglycemia-induced neuronal damage prevented by an $\mathrm{N}$ methyl-D-aspartate antagonist. Science 1985;230:681-3.
24 Greenamyre JT. The role of glutamate in neurotransmission and in neurologic disease. Arch Neurol 1986;43:1058.

25 Sugimoto K, Baba M, Suda T, et al. Peripheral neuropathy and microangiopathy in rats with insulinoma: association with chronic hyperinsulinemia. Diabetes Metab Res Rev 2003;19:392-400.

26 Savage S, Estacio RO, Jeffers B, et al. Increased complications in noninsulindependent diabetic patients treated with insulin versus oral hypoglycemic agents: a population study. Proc Assoc Am Physicians 1997; 109:181-9.

27 Sidenius $\mathbf{P}$, Jakobsen J. Anterograde fast component of axonal transport during insulin-induced hypoglycemia in nondiabetic and diabetic rats. Diabetes 1987;36:853-8.

28 Mandelbaum JA, Felten DL, Westfall SG, et al. Neuropathic changes associated with insulin treatment of diabetic rats: electron microscopic and morphometric analysis. Brain Res Bull 1983;10:377-84.

29 Hata A. Effect of nitric oxide on sciatic nerve blood flow in acute hypoglycemic rats. St Marianna Med 1996;24:370.

30 Kihara M, Zollman PJ, Smithson IL, et al. Hypoxic effect of exogenous insulin on normal and diabetic peripheral nerves. Am J Physiol 1994;266:E980-5.

31 Carmeliet P, Dor Y, Herbert JM, et al. Role of HIF-1 alpha in hypoxia-mediated apoptosis, cell proliferation and tumour angiogenesis. Nature 1998;394:485-90.

32 Halterman MW, Miller CC, Federoff HJ. Hypoxia-inducible factor-lalpha mediates hypoxia-induced delayed neuronal death that involves p53. J Neurosci 1999;19:6818-24

33 Brusselmans K, Bono F, Maxwell P, et al. Hypoxia-inducible factor-2alpha (HIF-2alpha) is involved in the apoptotic response to hypoglycemia but not to hypoxia. J Biol Chem 2001;276:39192-6.

34 Bastron JA, Lambert EH, Mulder DW. Hyperinsulin neuropathy. Neurology 1956:6:627-35.

35 Danta G. Hypoglycemic peripheral neuropathy. Arch Neurol 1969;21:121-32.

36 Harrison MJ. Muscle wasting after prolonged hypoglycemic coma. Case report with electrophysiological data. J Neurol Neurosurg Psychiatry 1976;39:465-70

37 Mohseni S. Hypoglycemic neuropathy. Acta Neuropathol 2001;102:413-21.

38 Puri V, Garg N, Kumar N, et al. Hypoglycemic neuropathy: a case report. Neurol India 2000:48:263-5. 